\title{
ON THE SPECTRUM OF TURBULENT CONVECTION
}

\author{
P. Ledoux, ${ }^{*}$ M. Schwarzschild, and E. A. SPIEgel \\ Princeton University Observatory, Princeton, New Jersey \\ Received July 21, 1960
}

\section{ABSTRACT}

A procedure is described by which, under certain assumptions, the turbulence spectrum can be derived for the motions in a convectively unstable layer. The energy input from the buoyancy forces is assessed in this procedure by deriving the growth rates of the laminar modes obtained from the relevant linearized equations On the other hand, the exchange of energy between modes is assumed to follow Heisenberg's elementary theory of turbulence. The procedure is carried through for an exceptionally simple case for which a closed solution was found for the spectrum. The results, though not strictly applicable, are applied to the convection in the solar photosphere for purposes of orientation.

\section{INTRODUCTION}

It now appears probable that the convection in the envelopes of stars of medium and late spectral type is of decisive importance for the determination of the radii of such stars, for the development of the outermost layers such as the solar chromosphere and corona, and possibly as a cause of the mass ejection from red giants. Accordingly, a theory of turbulent convection in stellar envelopes, more detailed and accurate than that now available, seems needed.

One of the main difficulties in the development of such a theory arises from the circumstance that the physical state varies greatly from the top to the bottom of a convective envelope of a star. Recent investigations (Skumanich 1955; Goody 1956; Gribov and Gurevich 1957; Unno 1957; Spiegel 1958, 1960; Boehm and Richter 1959) have attacked this difficulty by the use of laminar convection modes derived by first-order theory. Even though first-order theory can by itself clearly not lead to a full quantitative solution for turbulent convection, these investigations seem already to have produced some increased insight as to which of the physical characteristics of the envelope are the decisive ones for the convection.

Recently an extensive attack on the problem of fully turbulent convection was made by Malkus (1954). In his work the non-linear interactions between modes are not studied explicitly; instead, a statistical hypothesis regarding the relative energies in the various modes is introduced.

The question arises whether improvements in detailed insight and quantitative results can be achieved by combining the laminar solutions of first-order theory with the best available approximations for the effects of the quadratic interactions derived from present turbulence theory. In the present investigation this question is studied by taking the effect of the non-linear interactions to be represented by Heisenberg's (1948) explicit, approximate formula. This formula has already been applied by Parker (1951) in a similar connection; Parker's application, however, did not fully exploit the laminar solutions of first-order theory. It is clear that the present investigation does not represent a fundamental theory of turbulent convection, since it uses an approximate theory for the non-linear interactions.

\section{BASIC EQUATION}

The procedure adopted here runs as follows: Assume that you know the mean values (averaged in time or over a horizontal surface) for all relevant physical characteristics

\footnotetext{
* On leave from Institut d'Astrophysique, Liège, Belgium.
} 
throughout the convection zone; particularly assume that you know the mean density and the mean temperature gradient as a function of depth from the top to the bottom of the zone. Write the equation of motion (including buoyancy and viscosity terms), the continuity equation, and the heat equation (including the radiative conduction) in first-order approximation. Derive from these equations all possible laminar modes which fulfil the relevant boundary conditions. These modes will, in general, make up a full set of functions, so that an arbitrary field of turbulent velocities and temperature fluctuations can be represented by a linear combination of these modes. Accordingly, represent the stationary statistical state of turbulence in a stellar convection zone by determining the mean energy (average over time) contained in each mode. Determine this mean energy for each mode by considering all physical processes by which the mode in question can gain or lose energy. These processes are, first, the energy gain from the buoyancy force; second, the reduction of this energy gain by heat conduction; third, the energy loss by viscosity; and, fourth, energy gains or losses by dynamic interaction with other modes. The first three of these processes are automatically included in the first-order theory. Their net effect is represented by the growth rate, $n$, which follows for each mode directly from the solution of the first-order equations. The average rate of gain of energy from these three processes is therefore obtained for each mode by multiplying the average energy of this mode by $2 n$, where the factor 2 arises from the fact that the energy is a quadratic function of the amplitudes. This rate of energy gain will generally be positive for large-scale modes in which the buoyancy input overcomes the viscous dissipation but will be negative for small-scale modes in which the viscous effects dominate.

The fourth process-the dynamical interaction between modes-is by its nature a quadratic effect and can therefore not be taken account of in the first-order theory for the laminar modes. A quantitative estimate of the effects of these interactions has been given by Heisenberg (1952) in his elementary theory of turbulence. Since this theory is formulated in terms of the turbulence spectrum, the present procedure should continue as follows.

Assign to each laminar mode a total wave number, $k$. Sort all modes in order of $k$. Represent the average kinetic energy of all modes with total wave numbers between $k$ and $k+d k$ by $F(k) d k$ : thus $F(k)$ is the turbulence spectrum and has, as usual, the dimensions of velocity squared times length. Now form an appropriate average of the growth rates of all modes with the same total wave number $k$, and by this means define an effective growth rate $n(k)$ as a smooth function of $k$. With the help of this growth-rate function, the rate of energy gain by the three first-order processes of all modes with total wave numbers between a minimum wave number $k_{0}$ and $k$ can be expressed by

$$
E_{\mathrm{gain}}=\int_{k_{0}}^{k} F\left(k^{\prime}\right) 2 n\left(k^{\prime}\right) d k^{\prime} \text {. }
$$

On the other hand, according to Heisenberg (1948), the rate of energy loss of the same modes by quadratic interactions with all modes of higher wave number is given by

$$
E_{\mathrm{loss}}=2 \eta \int_{k}^{\infty}\left[\frac{F\left(k^{\prime \prime}\right)}{k^{\prime \prime 3}}\right]^{1 / 2} d k^{\prime \prime} \int_{k_{0}}^{k} F\left(k^{\prime}\right) k^{\prime 2} d k^{\prime}
$$

where $\eta$ is a non-dimensional factor of order unity.

In the stationary case the energy gains of equation (1) must exactly compensate the energy losses of equation (2), so that the condition for stationarity is given by

$$
\int_{k_{0}}^{k} F\left(k^{\prime}\right) 2 n\left(k^{\prime}\right) d k^{\prime}=2 \eta \int_{k}^{\infty}\left[\frac{F\left(k^{\prime \prime}\right)}{k^{\prime \prime 3}}\right]^{1 / 2} d k^{\prime} \int_{k_{0}}^{k} F\left(k^{\prime}\right) k^{\prime 2} d k
$$


Equation (3) is the central equation of the present investigation. Its derivation suggests that it may be applied without too serious errors to cases of moderately anisotropic turbulence, such as the solar photosphere. For any case for which the growth-rate function $n(k)$ is given, equation (3) should uniquely determine the spectrum $F(k)$, not only in its dependence on $k$ but also in its absolute value, since $F$ enters equation (3) inhomogeneously.

In the following sections equation (3) is applied exclusively to the most simple case of turbulent convection, namely, that of a layer in which the temperature gradient is constant in first approximation and in which the variations in density and temperature are so small that the classical Boussinesq approximation can be used. This oversimplified application in a sense defeats the purpose of the investigation, since the use of laminar modes was introduced expressly with the aim of handling cases with complicated temperature and density distributions such as occur in stellar envelopes. Nevertheless, it seems worthwhile to begin by studying this simplest case first.

In Section III the laminar modes and their growth rates are derived and discussed specifically for the range in Rayleigh number and Prandtl number relevant for the solar photosphere. In Section IV the basic equation as applied to the simple case is rederived from a more statistical point of view. The solution of the basic equation (3) for the simple case is developed in Section V and applied to the solar photosphere in Section VI.

\section{LAMINAR GROWTH RATES}

In accordance with the preceding section, we shall now consider the simplest possible case of a convectively unstable layer. Thus we shall assume the Boussinesq approximation to be applicable, i.e., the density and the temperature gradients to be negligible except where they enter the buoyancy term. Furthermore, again for simplicity, we shall assume the boundary conditions appropriate for so-called free boundaries.

Let $a=1 / T$ be the thermal expansion coefficient, $\beta=-\Delta \nabla T$ the temperature gradient excess over the adiabatic gradient, $\kappa$ the thermometric conductivity, $\nu$ the kinematic viscosity, $d$ the depth of the layer, $z$ the vertical co-ordinate, $u, v$, and $w$ the velocity components, $T^{\prime}$ the temperature perturbation, and $P^{\prime}$ the pressure perturbation. Then the classical solution for the principal modes of convection is represented by

$$
\begin{aligned}
u & =+V \frac{k_{z} k_{x}}{k^{2}} \cos \left(k_{x} x\right) \sin \left(k_{y} y\right) \cos \left(k_{z} z\right) e^{n t}, \\
v & =+V \frac{k_{z} k_{y}}{k^{2}} \sin \left(k_{x} x\right) \cos \left(k_{y} y\right) \cos \left(k_{z} z\right) e^{n t}, \\
w & =+V \frac{k_{x}^{2}+k_{y}^{2}}{k^{2}} \sin \left(k_{x} x\right) \sin \left(k_{y} y\right) \sin \left(k_{z} z\right) e^{n t}, \\
T^{\prime} & =+V \frac{n+\nu k^{2}}{g a} \sin \left(k_{x} x\right) \sin \left(k_{y} y\right) \sin \left(k_{z} z\right) e^{n t}, \\
P^{\prime} & =-V \frac{n+\nu k^{2}}{k} \frac{k_{z}}{k} \sin \left(k_{x} x\right) \sin \left(k_{y} y\right) \cos \left(k_{z} z\right) e^{n t} .
\end{aligned}
$$

Here the total wave number, $k$, is given by

$$
k^{2}=k_{x}^{2}+k_{y}^{2}+k_{z}^{2}
$$

and, according to the boundary conditions, the vertical wave number, $k_{z}$, is given by

$$
k_{z}=(N+1) \frac{\pi}{d}
$$


where $N$ represents the number of nodes in the vertical dimension. Finally-and most important for the present purpose- the growth rate for these principal modes is determined by the following characteristic equation:

$$
n=-\frac{\kappa+\nu}{2} k^{2}\left[1 \pm\left(1-\mu+\mu \frac{R}{k^{4} d^{4}} \frac{k_{x}^{2}+k_{y}^{2}}{k^{2}}\right)^{1 / 2}\right]
$$

where the parameter $\mu$ is defined in terms of the Prandtl number, $\sigma$, by

$$
\mu=\frac{4 \nu \kappa}{(\kappa+\nu)^{2}}=\frac{4 \sigma}{(1+\sigma)^{2}} \quad \text { with } \quad \sigma=\frac{\nu}{\kappa} .
$$

and $R$ stands for the Rayleigh number, defined by

$$
R=\frac{g a \beta d^{4}}{\kappa \nu}
$$

Equation (4) represents two types of modes in accordance with the two signs occurring in equation (7), the plus sign giving stable modes and the minus sign unstable modes. The unstable modes correspond to the usual convection patterns, while the stable modes correspond to hot elements moving downward. The unstable modes convect thermal energy upward and thus gain energy from the mean field. The stable modes convect energy downward and thus return their energy to the mean field.

In addition to the two types of modes given by equation (4), the equations of motion permit one more type of mode in which only horizontal velocities occur. This mode can be represented by

$$
\begin{aligned}
& u=+V \frac{k_{y}}{k} \cos \left(k_{x} x\right) \sin \left(k_{y} y\right) \cos \left(k_{z} z\right) e^{n t}, \\
& v=-V \frac{k_{x}}{k} \sin \left(k_{x} x\right) \cos \left(k_{y} y\right) \cos \left(k_{z} z\right) e^{n t}, \\
& w=T^{\prime}=P^{\prime}=0,
\end{aligned}
$$

where the growth rate is determined by

$$
n=-\nu k^{2}
$$

The three modes described by equations (4) and (10) together can represent an arbitrary field of $v, w$, and $T^{\prime}$ by an appropriate choice of the amplitude factors $V$. Under the Boussinesq approximation, $u$ and $P^{\prime}$ are not free functions when $v$, $w$, and $T^{\prime}$ are given, since under these conditions the continuity equation fixes $u$ and the divergence of the equation of motion fixes $P^{\prime}$. Hence the three types of modes given by equations (4) and (10) represent a complete set of functions in terms of which any field of velocities and of temperature and pressure fluctuations permissible under the present assumptions can be expressed.

If we now apply these laminar solutions, particularly their growth rates, to the solar photosphere, an appreciable simplification can be introduced into equation (7). Whenever a case of turbulent convection is considered, the Rayleigh number $R$ will be very large. Furthermore, in most laboratory cases, as well as in the stellar interior, the Prandtl number $\sigma$ will not be very small compared to 1 , and accordingly the parameter $\mu$ will be of order unity. Therefore, in these normal cases the last term in the parentheses of equation (7) will tend to be very large and will become of order unity only for very high wave numbers. In the solar atmosphere, however, the thermometric conductivity $\kappa$ is dominated by radiative conduction and thus turns out very large in comparison with the kinematic viscosity $\mu$. Thus the Prandtl number $\sigma$ and, in consequence, the 
parameter $\mu$ are very small in the solar photosphere. Indeed, if one introduces the numerical values appropriate for the solar photosphere (see Sec. VI) into equation (7), one finds that the last term in the parentheses of this equation is less than 1 even for the lowest wave numbers in question. Hence one may develop the root in equation (7), retain only linear terms, and still maintain reasonable accuracy. With this approximation for the solar photosphere, one finds, for the growth rates of the stable and the unstable modes, respectively,

$$
\begin{aligned}
& n_{s}=-\kappa k^{2}-\frac{g a \beta}{\kappa} \frac{k_{x}^{2}+k_{y}^{2}}{k^{2}} \frac{1}{k^{2}}, \\
& n_{u}=-\nu k^{2}+\frac{g a \beta}{\kappa} \frac{k_{x}^{2}+k_{y}^{2}}{k^{2}} \frac{1}{k^{2}} .
\end{aligned}
$$

Equations (11), (12), and (13) give the growth rates of each of the three types of modes - the horizontal ones, the stable ones, and the unstable ones-each as a function of the wave number $k$. We shall now proceed to indicate that probably only the unstable modes are of importance for the present purpose, so that the growth rates for the horizontal modes and the stable modes may be ignored.

As shown by equation (11), the horizontal modes are not excited by the buoyancy force and therefore contribute probably little, in comparison with the unstable mode, to the energy spectrum $F(k)$ at the lower wave numbers. At the higher wave numbers, where the energy in the horizontal modes may be built up by the coupling terms with the unstable modes, the negative growth rate, i.e., the viscous dissipation, of the horizontal modes is just equal to that of the unstable modes, as shown by a comparison of equations (11) and (13). Accordingly, it would seem a permissible approximation if equation (13) is taken to represent the horizontal modes also.

Regarding the stable modes, equation (12), in conjunction with the numerical values for the solar photosphere given in Section VI, shows that their negative growth rate, i.e., their effective dissipation, is very large and, indeed, exceeds the positive growth rate of the unstable modes even for the lowest wave numbers in question. Thus it seems likely that in the solar photosphere, owing to the very high radiative thermometric conductivity, the stable modes are extremely highly damped and may possess negligible energy in comparison with the unstable modes. Altogether, therefore, we shall accept equation (13) as representing the growth-rate function for the entire assembly of modes in the solar photosphere.

We still have to consider the relation between the horizontal wave numbers and the total wave number, the ratio of which occurs in equation (13). The fundamental equations and the boundary conditions used here admit only a discrete set of vertical wave numbers $k_{z}$ in accordance with equation (6). But for each vertical wave number the whole continuum of horizontal wave numbers is admitted. However, for a given vertical wave number there exists one specific horizontal wave number which gives the mode with the highest growth rate according to equation (13). For these maximum-rate modes the relations between the horizontal wave number, the vertical wave number, and the total wave number are approximately

$$
k_{x}^{2}+k_{y}^{2}=k_{z}^{2}=\frac{1}{2} k^{2}
$$

We shall use this relation as representative of all the essential modes containing appreciable energy. Accordingly, equation (13) takes the simple form

$$
n(k)=-\nu k^{2}+\frac{g a \beta}{2 \kappa} \frac{1}{k^{2}} .
$$


Throughout the subsequent sections we shall use equation (15) as giving the growth rate for all modes in the solar photosphere as a function of the wave number $k$. For small wave numbers, the second term in equation (15) is clearly dominant; it determines the rate of energy gain of a mode by the buoyancy force. On the other hand, for very large wave numbers, the first term in equation (15) is dominant; it determines the energy dissipation rate by viscosity. Between the low wave numbers at which the energy input by the buoyancy force occurs and the very high wave numbers at which the viscous dissipation occurs, there exists in the solar photosphere a very large range in wave numbers in which neither of these two processes is effective, i.e., in which equation (15) gives practically zero growth rates.

Finally, a minimum total wave number has still to be fixed. The minimum admissible vertical wave number $k_{z}$ is determined by equation (6) with $N=0$. If this value for the minimum vertical wave number is introduced into relation (14), one obtains a minimum total wave number given by

$$
k_{0}=\sqrt{ } 2 \frac{\pi}{d}
$$

With the help of the growth-rate function of equation (15) and the minimum wave number of equation (16) we can now attempt to determine the turbulence spectrum for the case of low Prandtl number from the basic equation (3). This will be undertaken in Section V. But first we shall discuss the present problem from a more statistical point of view in the next section.

\section{STATISTICAL DERIVATION OF THE BASIC EQUATION}

In Section II we describe the energy balance in steady-state convection by equating the laminar input over a range of wave numbers to the non-linear output in the same range. With simplified descriptions of both the laminar and the non-linear terms, we summarized this balance by equation (3). We shall now rederive equation (3) from the equations of motion, in order to clarify the approximations which underlie our simplified description.

As mentioned previously, we shall restrict our considerations to convection in a layer whose thickness is much less than its scale height. The equations governing the motion are, then (cf. Spiegel and Veronis 1960),

$$
\begin{gathered}
\nabla \cdot v=0 \\
\frac{\partial v}{\partial t}+v \cdot \nabla v=-\frac{1}{\rho} \nabla P^{\prime}+\nu \nabla^{2} v+g a T^{\prime} \lambda \\
\frac{\partial T^{\prime}}{\partial t}+v \cdot \nabla T^{\prime}=\kappa \nabla^{2} T^{\prime}+\beta v \cdot \lambda
\end{gathered}
$$

where $\lambda$ is a unit vector in the vertical direction, primes denote fluctuation quantities, and all other quantities (except $v$ ) are averaged over the fluid. In particular, $\beta$ is taken to be constant in the present treatment, though this will not be the case in higher approximations. Equation (19) contains the assumption that all lengths of interest are optically thick, so that the radiative term may be represented by a simple conductivity.

Our procedure will now be to make a Fourier resolution of the turbulent field, though ideally we should expand in the normal modes of the linearized system. However, since we intend to deal here with the case in which only the unstable modes (see Sec. III) are important, the Fourier mode for each wave number should be almost a pure unstable mode, and our present procedure should suffice. 
Let us then consider a volume occupying the full height, $d$, of the convecting layer and having a square horizontal cross-section of dimension $L$. Over this volume we may represent the fluctuating quantities in Fourier series as, for example,

$$
v(x, t)=\sum_{k} u(k, t) e^{i k \cdot x}
$$

and

$$
T^{\prime}(x, t)=\sum_{k} \theta(k, t) e^{i k \cdot x}
$$

Here $\boldsymbol{u}$ and $\theta$ are the usual Fourier amplitudes, and the summations are over all $\boldsymbol{k}$ consistent with the free-boundary conditions set forth in Section II.

We may then, as is normally done, deduce equations for the power spectra from the equations of motion. A useful approximation for us will be to replace sums over $k$ by integrals. This is quite adequate for sums over horizontal components of $k$, since we may let $L \rightarrow \infty$; but for sums over vertical $k$-components this is a significant approximation.

Also, though we are not dealing with isotropic turbulence, we should like to characterize the turbulent energy as a function of the scalar wave number. To effect such a description, we shall integrate the equations over a spherical surface in $k$-space. The power spectra with which we then have to deal are

and

$$
F(k)=\frac{L^{2} d}{8 \pi^{3}} k^{2} \int\left\langle\boldsymbol{u} \cdot \boldsymbol{u}^{*}\right\rangle d \Omega
$$

$$
G(k)=\frac{L^{2} d}{8 \pi^{3}} k^{2} \int\left\langle\theta \theta^{*}\right\rangle d \Omega .
$$

The brackets denote ensemble averages.

To complete our description, we also need the spectrum of the velocity-temperature correlation:

$$
H(k)=\frac{1}{2} \frac{L^{2} d}{8 \pi^{3}} k^{2} \lambda \int\left\langle\boldsymbol{u} \theta^{*}+\boldsymbol{u}^{*} \theta\right\rangle d \Omega .
$$

The derivation of the equations for the spectra from equations (18) and (19) is then standard, except for the inclusion of the buoyancy term. The resulting equations for the steady state are

$$
\int_{k_{0}}^{\infty} Q\left(k, k^{\prime}\right) d k^{\prime}=2 \nu k^{2} F-2 g a H
$$

and

$$
\int_{k_{0}}^{\infty} U\left(k, k^{\prime}\right) d k^{\prime}=2 \kappa k^{2} G-2 \beta H .
$$

In these equations the integral terms result from the non-linear terms of equations (18) and (19); $Q$ is trilinear in velocity, and $u$ is bilinear in temperature and linear in velocity. The cutoff wave number $k_{0}$ has been discussed in Section II.

Assuming that we know the structure of the non-linear coupling terms, equations (25) and (26) are two equations in the three unknowns, $F, G$, and $H$. To make the system determinate, we must add an equation which specifies the phase relations between $u$ and $\theta$. In the present case, since the unstable modes predominate in the spectrum at each wave number (see Sec. II), we may assume a phase relationship characteristic of the unstable modes. That is, we may assume that $u$ and $\theta$ are completely in phase and, hence, that $H$ is proportional to $\sqrt{ }(F G)$. 
Now $F$ gives twice the total kinetic energy per wave number interval, and we want only that part associated with vertical motion. We might, for example, assume isotropy and take $\frac{1}{3} F$ as the vertical contribution. However, to stay in accord with Section III, we shall use here the flow pattern of the most unstable mode. Thus, using equations (4) and (14), we conclude that twice the kinetic energy of vertical motion is $\frac{1}{2} F$. Then our assumption that velocity and temperature are in phase may be expressed by

$$
H=\sqrt{ }\left(\frac{1}{2} F G\right) \text {. }
$$

In addition to the specification of phases, we shall introduce another simplifying restriction. In stellar atmospheres the radiative conductivity is quite high, and there are probably many cases of interest where the effective turbulent conductivity is considerably less. The condition for the neglect of the turbulent conductivity is that the ratio of the non-linear term to the conduction term in equation (19) should be small. This ratio is measured by the Peclet number, $v d / \kappa$, where $v$ is a characteristic speed. At the top of the solar convective zone, for example, the Peclet number (which is equivalent to the quantity $\Gamma$ in the work of Vitense 1952) is close to unity. Under these circumstances, probably no great error is made if the turbulent conduction, i.e., the non-linear term in equation (19) and hence the integral term in equation (26), is neglected. With this additional simplification, equation (26) becomes

$$
\kappa k^{2} G=\beta H .
$$

If we then eliminate $H$ between equations (27) and (28), we obtain

$$
G=\frac{\beta^{2}}{2 \kappa^{2}} \cdot \frac{1}{k^{4}} F
$$

This relation between $G$ and $F$ might also be derived directly from equations (4) and (14).

Now, on combining equations (25) and (28), we obtain an equation for $F$, namely,

$$
2\left(\frac{g a \beta}{2 \kappa} \frac{1}{k^{2}}-\nu k^{2}\right) F=-\int_{k_{0}}^{\infty} Q\left(k, k^{\prime}\right) d k^{\prime} .
$$

We see that the coefficient of $F$ on the left-hand side is just $2 n$, where $n$ is given by expression (15). If we then integrate equation (30) from $k_{0}$ to $k$ (where $k_{0}$ is the cutoff wave number discussed in Sec. II) and apply Heisenberg's (1948) estimate for the righthand side, we obtain equation (3). In the next section, we proceed with the solution of this equation.

\section{SOLUTION OF THE BASIC EQUATION}

If one introduces equation (15) for the growth rate into the central equation (3), the latter takes the form

$$
\int_{k_{0}}^{k} F\left(k^{\prime}\right) \frac{g a \beta}{\kappa} \frac{1}{k^{\prime 2}} d k^{\prime}=2\left\{\nu+\eta \int_{k}^{\infty}\left[\frac{F\left(k^{\prime}\right)}{k^{\prime 3}}\right]^{1 / 2} d k^{\prime}\right\} \int_{k_{0}}^{k} F\left(k^{\prime \prime}\right) k^{\prime \prime 2} d k^{\prime \prime} .
$$

A procedure for solving equation (31) is suggested by Chandrasekhar's (1949) solution of the Heisenberg (1948) equation for steady, isotropic turbulence. Before carrying out the solution, however, let us introduce a non-dimensional spectrum, $f$, and wave number, $q$, by the relations

and

$$
F=\left(\frac{g a \beta}{2 \eta \kappa}\right)^{2} k_{0}^{-7} f
$$

$$
k=k_{0} q \text {. }
$$


Equation (31) becomes

Here

$$
\int_{1}^{q} \frac{f\left(q^{\prime}\right)}{q^{\prime 2}} d q^{\prime}=\left\{\delta+\int_{q}^{\infty}\left[\frac{f\left(q^{\prime \prime}\right)}{q^{\prime \prime 3}}\right]^{1 / 2} d q^{\prime \prime}\right\} \int_{1}^{q} q^{\prime 2} f\left(q^{\prime}\right) d q^{\prime} .
$$

$$
\delta=\frac{8 \pi^{4}}{R}
$$

and the Rayleigh number, $R$, is defined in equation (9).

Differentiation of equation (34) leads to the equation

$$
\delta-\frac{1}{q^{4}}+\int_{q}^{\infty}\left[\frac{f\left(q^{\prime \prime}\right)}{q^{\prime \prime 3}}\right]^{1 / 2} d q^{\prime \prime}-\left(f q^{7}\right)^{-1 / 2} \int_{1}^{q} q^{\prime 2} f\left(q^{\prime}\right) d q^{\prime}=0 .
$$

To simplify this equation, we introduce the transformations

and

$$
g=f q^{7}
$$

$$
y=\int_{1}^{q} q^{\prime 2} f\left(q^{\prime}\right) d q^{\prime} \text {. }
$$

Differentiation of the transformed equation with respect to $y$ then produces the differential equation,

$$
\frac{d g}{d y}+\frac{8 \sqrt{ } g}{y}-\frac{4 g}{y}=0 \text {. }
$$

The general solution of this equation is

$$
g=\left(A y^{2}+2\right)^{2},
$$

where $A$ is an integration constant to be determined below.

To complete the solution, we must explicitly relate $y$ and $q$. By differentiating equation (38) and making use of equation (37), we obtain another differential equation,

$$
\frac{d q}{q^{5}}=\frac{d y}{g}
$$

Since we know $g$ as a function of $y$, we can integrate this equation to obtain

$$
q^{4}=\left[1-\frac{y}{A y^{2}+2}-\frac{1}{\sqrt{ }(2 A)} \arctan \sqrt{ }\left(\frac{A y^{2}}{2}\right)\right]^{-1}
$$

where only principal values of arctan are to be taken.

This completes the solution of the basic equation, and we may summarize our result in the following equation:

$$
f=4 q^{-7}\left(x^{2}+1\right)^{2}
$$

where $x=\sqrt{ }(A / 2) y$ is given by

$$
q^{-4}=1-\frac{1}{\sqrt{ }(2 A)}\left(\frac{x}{x^{2}+1}+\arctan x\right) .
$$

Hence $f$ is found as an implicit function of $q$, and it remains only to evaluate $A$.

From equation (34) applied to $q=\infty$, we obtain, with the help of equations (37) and (41),

$$
\int_{0}^{y_{\infty}} \frac{d y}{q^{4}}=y_{\infty} \delta
$$


where $y_{\infty}$ is the value of $y$ at $q=\infty$. Using equation (42), we may carry out the required integration, with the following result:

$$
y_{\infty}=\sqrt{ }\left(\frac{2}{A}\right) \tan [\sqrt{ }(2 A)(1-\delta)]
$$

A second relation between $y_{\infty}$ and $A$ may be deduced from equation (42). With this we can eliminate $y_{\infty}$ from equation (46) and determine the following condition on $A$ :

$$
\sin [2 \sqrt{ }(2 A)(1-\delta)]=2 \sqrt{ }(2 A) \delta .
$$

Disregarding the extraneous root $A=0$, we see that $A$ goes from $\pi^{2} / 8$ to zero as $\delta$ goes from 0 to $\frac{1}{2}$. We are interested primarily in small $\delta$, in which case $A$ is approximated by

$$
A=\frac{\pi^{2}}{8}\left(1-\frac{\pi^{2}}{3} \delta^{3}\right)
$$

The unique solution defined by equations (43), (44), and (48) is represented in Table 1 and Figures 1 and 2 for $\delta=0$, i.e., $R=\infty$.

TABLE 1

\begin{tabular}{|c|c|c|c|c|c|c|c|}
\hline$x$ & $q$ & $f$ & $f / q^{4}$ & $x$ & $q$ & $f$ & $f / q^{4}$ \\
\hline $\begin{array}{ll}0 & 00 \\
0 & 25 \\
0 & 50 \\
0 & 75 \\
1 & 00\end{array}$ & $\begin{array}{ll}1 & 000 \\
1 & 096 \\
1 & 221 \\
1 & 369 \\
1 & 532\end{array}$ & $\begin{array}{ll}4 & 00 \\
2 & 38 \\
1 & 55 \\
1 & 08 \\
0 & 81\end{array}$ & $\begin{array}{ll}4 & 00 \\
1 & 66 \\
0 & 70 \\
0 & 31 \\
0 & 15\end{array}$ & $\begin{array}{ll}1 & 50 \\
2 & 00 \\
2 & 50 \\
3 & 00\end{array}$ & $\begin{array}{ll}1 & 877 \\
2 & 229 \\
2 & 576 \\
2 & 916\end{array}$ & $\begin{array}{rr}0 & 51 \\
37 \\
\\
\\
028 \\
022\end{array}$ & $\begin{array}{rr}0 & 04 \\
01 \\
01 \\
0 & 00\end{array}$ \\
\hline
\end{tabular}

Velocity Spectrum, $f$, And Temperature Spectrum, $f / q^{4}$, AS Function of WAVE Number, $q$, FOR Simple CASE OF TURbUlent CONVECTION

With equation (48) for $A$ in the region of large $R$, we are in a position to examine our solution in various asymptotic limits. From equations (43) and (44) we learn that there are three main regions of interest:

and

$$
\begin{array}{ll}
\text { for } 1 \leq q \ll 1.25, & f \propto q^{-7} ; \\
\text { for } 1.25 \ll q \ll\left(\frac{R}{8 \pi^{4}}\right)^{3 / 4}, & f \propto q^{-5 / 3} ;
\end{array}
$$

$$
\text { for } q \gg\left(\frac{R}{8 \pi^{4}}\right)^{3 / 4}, \quad f \propto q^{-7} \text {. }
$$

Thus after a steep descent at the lowest wave number permitted by the boundary conditions, the spectrum makes an early transition to the familiar Kolmogoroff power law. As might be expected, the band of wave numbers in which Kolmogoroff's law holds increases as $R$ increases. Also, the final transition to the minus-seventh law is a natural result of our use of Heisenberg's representation for the non-linear term.

Clearly, the exact form of the spectra shown in Figures 1 and 2 for the low wave numbers is strongly determined by the approximations used for the lowest modes. It seems plausible, however, that the over-all distribution of power in the spectra is not seriously affected by these approximations.

To conclude this discussion of the mathematics, let us compute expressions for the root-mean-square velocity and temperature fluctuations averaged over the entire layer, 
as well as for the correlation between the vertical velocity and the temperature. With the help of transformations (32) and (33) we find, for the first of the quantities,

$$
\left\langle v^{2}\right\rangle=\int_{k_{0}}^{\infty} F(k) d k=\left(\frac{g a \beta}{2 \eta \kappa}\right)^{2} k_{0}^{-6} \int_{1}^{\infty} f(q) d q,
$$

Similarly, the second quantity follows either from equations (4) and (14) or from equation (29):

$$
\left\langle T^{2}\right\rangle=\int_{k_{0}}^{\infty} G(k) d k=\frac{\beta^{2}}{2 \kappa^{2}} \int_{k_{0}}^{\infty} \frac{F(k)}{k^{4}} d k=\frac{\beta^{2}}{2 \kappa^{2}}\left(\frac{g a \beta}{2 \eta \kappa}\right)^{2} k_{0}^{-10} \int_{1}^{\infty} \frac{f(q)}{q^{4}} d q .
$$

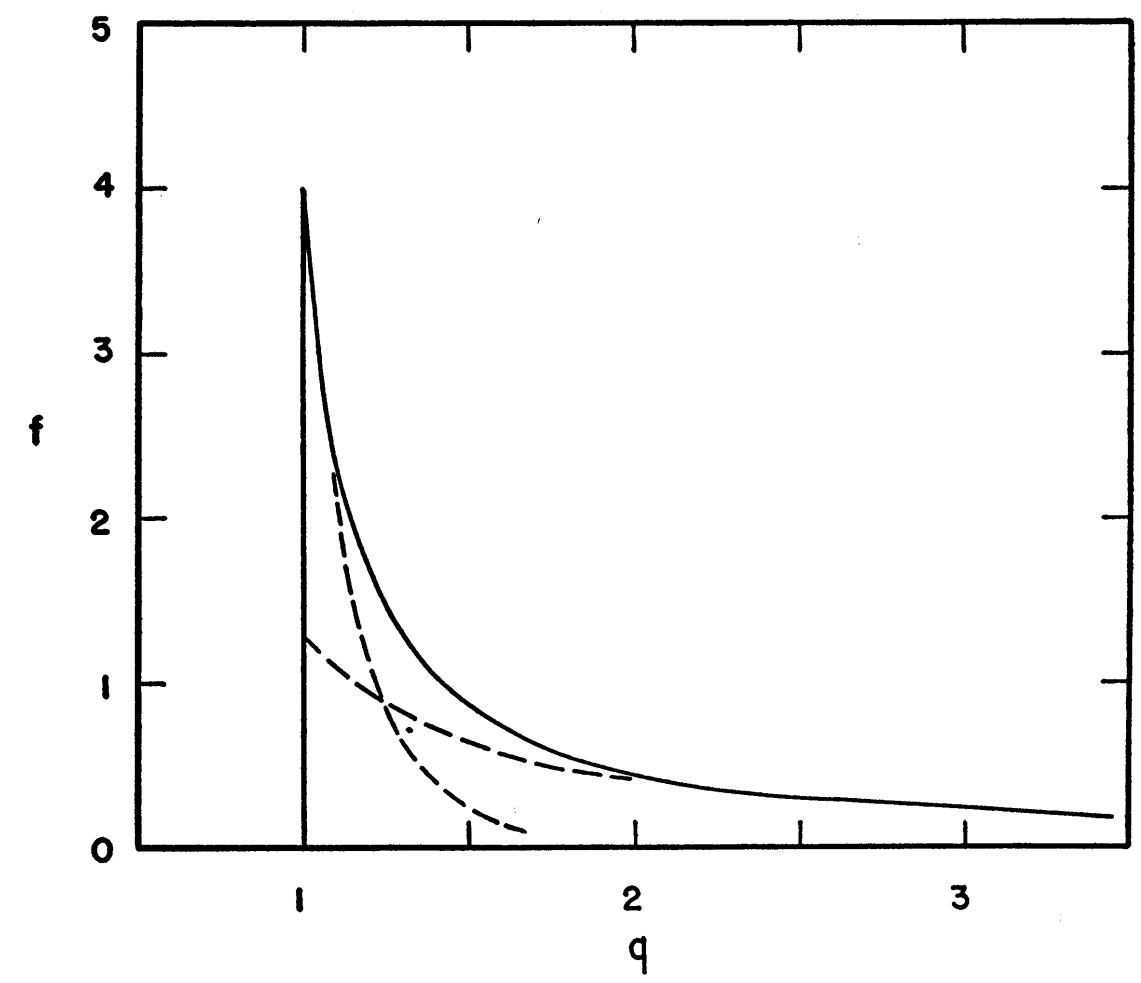

Frg. 1. - Velocity spectrum for simple case of convective zone. Steep dashed curve: limit for large eddies $\left(f=4 q^{-7}\right)$. Flat dashed curve: Kolmogoroff spectrum $\left(f=1.275 q^{-5 / 3}\right)$.

Finally, we find the third quantity either from equation (27) or from equations (4) and (14):

$$
\left\langle w T^{\prime}\right\rangle=\int_{k_{0}}^{\infty} H(k) d k=\frac{\beta}{2 \kappa} \int_{k_{0}}^{\infty} \frac{F(k)}{k^{2}} d k=\frac{\beta}{2 \kappa}\left(\frac{g a \beta}{2 \eta \kappa}\right)^{2} k_{0}^{-8} \int_{1}^{\infty} \frac{f(q)}{q^{2}} d q .
$$

The three integrals in these expressions have definite numerical values (for large $R$ ) which can be determined from the solution given in equations (43) and (44). If one introduces these numerical values, as well as equation (16) for the three preceding expressions, one finally obtains

$$
\begin{aligned}
& \left\langle v^{2}\right\rangle=\left(\frac{\kappa}{d}\right)^{2}\left(\frac{g a \beta d^{4}}{\kappa^{2} 8 \pi^{4}}\right)^{2} \frac{2 \pi^{2}}{\eta^{2}} \times 2.44, \\
& \left\langle T^{\prime 2}\right\rangle=(\beta d)^{2}\left(\frac{g a \beta d^{4}}{\kappa^{2} 8 \pi^{4}}\right)^{2} \frac{1}{4 \eta^{2} \pi^{2}} \times 0.524,
\end{aligned}
$$


and

$$
\left\langle w T^{\prime}\right\rangle=(\kappa \beta)\left(\frac{g a \beta d^{4}}{8 \pi^{4} \kappa^{2}}\right)^{2} \frac{1}{2 \eta^{2}} \times \frac{8}{\pi^{2}} .
$$

VI. APPLICATION TO SOLAR PHOTOSPHERE

The general procedure outlined in the first two sections has been carried through in detail in the three subsequent sections for a layer with a constant temperature gradient excess, constant viscosity and thermometric conductivity, and negligible density gradient. Furthermore, the investigation has been simplified by restricting it to the case of high Rayleigh number and low Prandtl number. The main results for this case are given in Figure 1 for the velocity spectrum, in Figure 2 for the temperature spectrum and, in equations (52), (53), and (54) for the statistical averages of the principle fluctuations.

In the solar photosphere it is true that the Rayleigh number is high and the Prandtl number low, but the mean density and particularly the conductivity are by no means constant. Nevertheless, it is tempting to apply the present results to the convection in

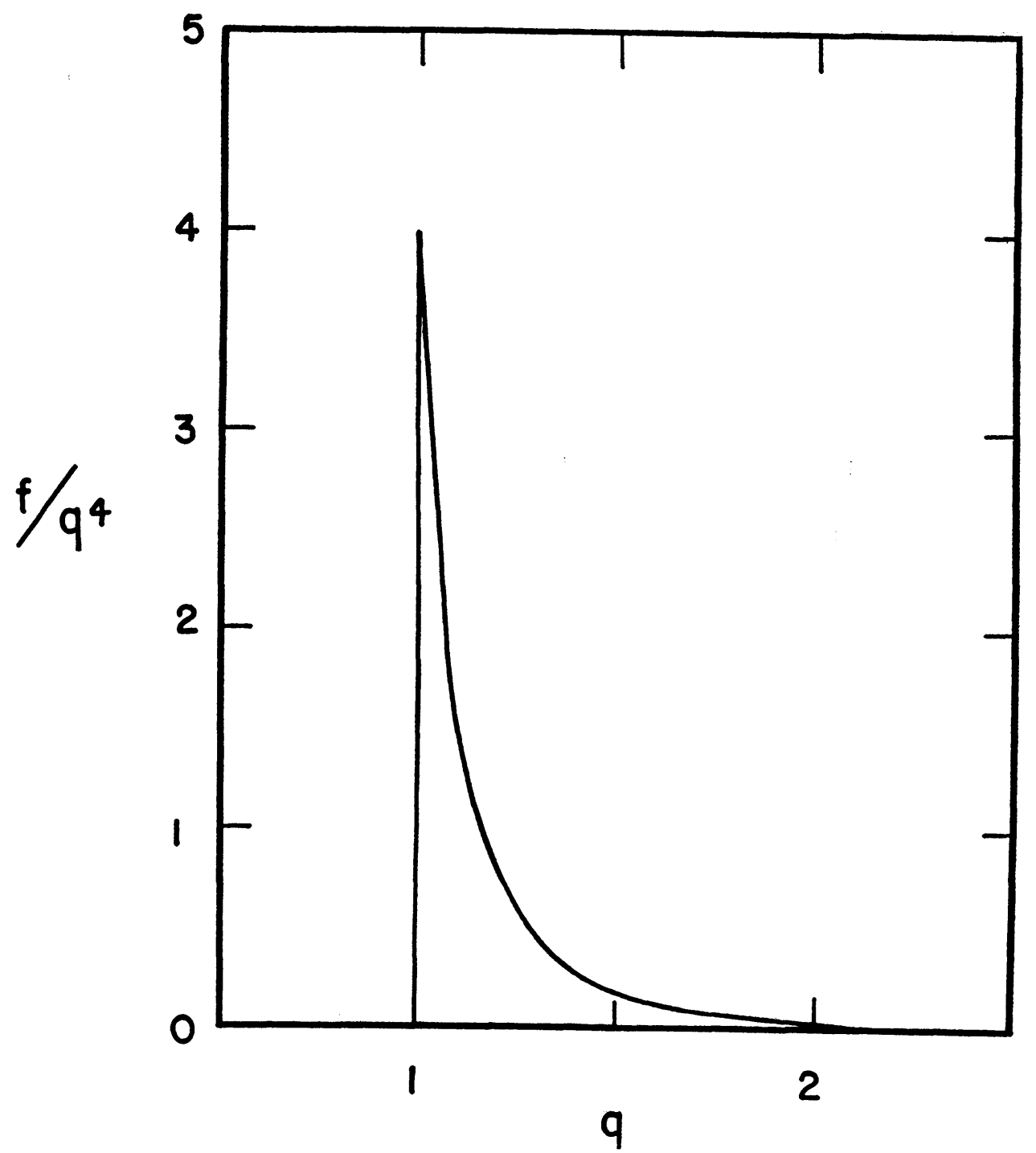

FIG. 2.-Temperature spectrum for simple case of convective zone 
the solar photosphere in order to orient one's self in the application of the procedure outlined here. We do this in the full realization that this application can be of only the coarsest kind and will likely not lead to new or more accurate results.

Table 2 gives the run of the relevant quantities with depth for the solar convection

TABLE 2

SOLAR CONVECTION ZONE

\begin{tabular}{|c|c|c|c|c|c|c|c|}
\hline$\underset{(\mathrm{km})}{Z}$ & $\log P$ & $T$ & $\rho \times 10^{7}$ & $\mu$ & $C_{p} \times 10^{8}$ & $\log \nu$ & $\log \kappa$ \\
\hline $\begin{array}{r}0 \\
100 \\
200 \\
300 \\
400 \\
500\end{array}$ & $\begin{array}{ll}4 & 97 \\
5 & 20 \\
5 & 41 \\
5 & 59 \\
5 & 75 \\
5 & 88\end{array}$ & $\begin{array}{r}6100 \\
8300 \\
10500 \\
11500 \\
12400 \\
13000\end{array}$ & $\begin{array}{ll}2 & 8 \\
3 & 5 \\
4 & 3 \\
5 & 5 \\
7 & \\
9 & \end{array}$ & $\begin{array}{ll}1 & 50 \\
1 & 49 \\
1 & 42 \\
1 & 32 \\
1 & 26 \\
1 & 22\end{array}$ & $\begin{array}{r}14 \\
24 \\
7 \\
9 \\
11 \\
12\end{array}$ & $\begin{array}{ll}3 & 3 \\
3 & 2 \\
3 & 1 \\
3 & 0 \\
2 & 9 \\
2 & 8\end{array}$ & $\begin{array}{rl}13 & 0 \\
11.8 \\
10 & 7 \\
10 & 1 \\
9 & 6 \\
9 & 3\end{array}$ \\
\hline
\end{tabular}

zone. These data represent roughly the average of the two solutions derived by Vitense (1953). The last two columns were read from the tables of Edmonds (1957). From these data we select the following mean values:

$$
\begin{array}{lll}
d=300 \mathrm{~km}, & a=\frac{1}{T}=10^{-4}, & \beta=-\Delta \nabla T=10^{-4}, \\
g=2.7 \times 10^{4} & \nu=10^{3} \mathrm{~cm}^{2} / \mathrm{sec}, & \kappa=10^{12} \mathrm{~cm}^{2} / \mathrm{sec} .
\end{array}
$$

The depth $d$ chosen here corresponds to approximately 1.5 pressure scale heights and a density change of about a factor of 2 . The value of $\beta$ corresponds to a superadiabatic temperature excess of $3000^{\circ}$ over the depth of $300 \mathrm{~km}$; this temperature excess was estimated from Figure 4 of Vitense (1953). The choice of the values of $d$ and particularly for $\kappa$ is clearly highly arbitrary, and this fact illustrates most directly the weakness of the application of the results for the simple case to the solar convection. The specific values chosen for $d$ and $\kappa$ were selected expressly with the aim of making the following results agree by order of magnitude with the observations.

With the help of the selected values given in equation (55) we find, for the Rayleigh number, the Prandtl number, and the combination of these quantities occurring in equations (52)-(54),

$$
\begin{aligned}
R & =\frac{g a \beta d^{4}}{\nu \kappa}=2 \times 10^{11}, \quad \sigma=\frac{\nu}{\kappa}=10^{-9}, \\
\frac{\sigma R}{8} \frac{g a \beta d^{4}}{\pi^{4}} & =\frac{g .3 .}{\kappa^{2} 8 \pi^{4}}=0 .
\end{aligned}
$$

If we further adopt for the Heisenberg coupling constant $\eta$ in equation (2) the laboratory result (Batchelor 1953),

$$
\eta \approx \frac{1}{3}
$$

we finally obtain from equations (52), (53), and (54)

$$
\begin{gathered}
\sqrt{ }\left\langle v^{2}\right\rangle=2 \mathrm{~km} / \mathrm{sec}, \quad\left\langle T^{\prime 2}\right\rangle=300^{\circ}, \\
\frac{\left\langle w T^{\prime}\right\rangle}{\kappa|\nabla T|}=0.4 \frac{\beta}{|\nabla T|} \approx 0.2 .
\end{gathered}
$$

Thus we obtain a value for the root-mean-square velocity (sum of all three components) which is about half the observed one (Waddell 1954), while the value for the root-mean- 
square temperature fluctuation we have obtained is about three times larger than the observed one (Schwarzschild 1959). The last item of equation (58) indicates that the convective energy transport should be of the same order of magnitude as the radiative transport, which agrees in a rough sense with previous theoretical estimates.

In view of the great arbitrariness in the application of the results for the simple case to the solar convection, the most we are permitted to conclude is that the theoretical results can be made to agree with the observations in order of magnitude by a not unreasonable choice of the relevant physical quantities.

There is one more item in which our present results may give useful suggestions for the interpretation of the solar convection. Figure 1 shows that about half the power of the velocity spectrum lies at $q$-values larger than 2 , i.e., in eddies with diameters smaller than half the diameter of the most unstable eddies. In contrast, Figure 2 shows that practically all the power in the temperature spectrum lies at $q$-values between 1 and 2 , i.e., in eddies only slightly smaller than the most unstable ones. If this feature is not altered in higher approximations and if the diameter of the most unstable eddies is about 600 $\mathrm{km}$, then the resolution of $300 \mathrm{~km}$ recently reached from stratospheric balloons should suffice to resolve the bulk of the temperature spectrum (and hence the surface intensity spectrum). However, with the same resolution in a spectrograph, perhaps only half the power of the velocity spectrum would be resolved. Furthermore, the existence of substantial kinetic energy in small eddies may greatly increase the production of progressive waves (be they hydromagnetic or hydrodynamic) capable of feeding energy into the corona.

If more definite results are to be obtained for the solar convection from the present procedure, the following steps are necessary: The relevant variation of the critical physical quantities have to be computed. The growth rates of the modes have to be derived and introduced into our basic equation (3). Equation (3) must then be solved anew. With the velocity and temperature spectra derived in this manner, the convective energy transport can be computed as a function of depth throughout the zone. Finally, the original model for the convection zone can be improved by the use of the condition that the total energy transport must be constant with depth. Such an iteration procedure would be similar to that frequently used in the construction of radiative model atmospheres.

Before embarking on the appreciable numerical work necessary to obtain more definite results for the solar convection by the present procedure, it would seem worthwhile to compute through in detail a specific convection case for which definite data are available from laboratory experiments (Prandtl number not far from 1), with the aim of thus testing the validity of the present procedure. An attempt to carry through such a test has been begun.

\section{REFERENCES}

Batchelor, G. K 1953, The Theory of Homogeneous Turbulence (Cambridge University Press), p. 167

Böhm, K. H., and Richter, E. 1959, Zs. f. A p., 48, 231.

Chandrasekhar, S 1949, Proc. R. Soc., London, A, 200, 20

Edmonds, F. N. 1957, Ap. J., 125, 535 .

Goody, R. M. 1956, J. Fluid Mech., 1, 424.

Gribov, V. N., and Gurevich, L. E.'1957, J. Exper. Theoret. Physics, 4, 720.

Heisenberg, W. 1948, Zs f. Phys., 124, 628.

Malkus, W. V. R. 1954, Proc. R. Soc. London, A, 225, 196.

Parker, E. N. 1951, Tech. Rep. No. IV, University of Utah.

Schwarzschild, M. 1959, Ap. J., 130, 345.

Skumanich, A. 1955, $A p$. J., 121, 408.

Spiegel, E A. 1958, dissertation, University of Michigan.

1960. Ap.J., in press.

Spiegel, E. A., and Veronis, G. 1960, Ap. J., 131, 442.

Unno, W. 1957, Ap. J., 126, 259.

Vitense, E. 1953, Zs. f. Ap., 32, 135.

Waddell, J. H., III, 1958, A p. J., 127, 284. 